Supplement of Geosci. Commun., 3, 365-379, 2020

https://doi.org/10.5194/gc-3-365-2020-supplement

(C) Author(s) 2020. This work is distributed under

the Creative Commons Attribution 4.0 License.

(c) (1)
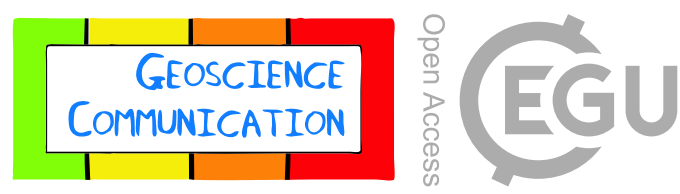

Supplement of

\title{
Developing the hertz art-science project to allow inaudible sounds of the Earth and cosmos to be experienced
}

\section{Graeme J. Marlton and Juliet Robson}

Correspondence to: Graeme J. Marlton (graeme.marlton@ reading.ac.uk)

The copyright of individual parts of the supplement might differ from the CC BY 4.0 License. 


\section{A1: Signal processing}

This section outlines how the signal processing for the final production run of hertz was undertaken.

Let us take an infrasonic times series $S(t)$ where $t$ is the time index of the infrasound recording which is recorded at $20-50 \mathrm{~Hz}$ dependent on infrasound sensor used. $S(t)$ is passed through a bandpass $5^{\text {th }}$ Order Butterworth filter. A band pass filter allows waveforms with frequencies between two given cut off frequencies to be passed and waveforms with frequencies outside that limit to be removed. The bandpass range of the $5^{\text {th }}$ order Butterworth filter is typically set between 0.1 and $10 \mathrm{~Hz}$, this varied dependent on the infrasound being processed see figures $3 \& 4$ in the main text.

This produces a band pass filtered signal $B P(t)$. To make this signal suitable for playback through audio equipment the sampling frequency needed increasing. This was undertaken by re-sampling $B P(t)$ at $44.1 \mathrm{kHz}$.

Next the re sampled band pass filtered signal $B P_{R S}(t)$ is normalised before being multiplied element wise by synthetically generated Pink Noise sampled at $44.1 \mathrm{kHz}$ to yield a new time series $\mathrm{X}(\mathrm{t})$ as shown in equation 1

$$
X(t)=\frac{B P_{R S}(t)}{\max \left(B P_{R S}\right)} P N(t)
$$

Where $P N(t)$ is a time series containing the synthetically generated Pink Noise. As the Pink Noise generated covers the whole audible frequency range a low pass $5^{\text {th }}$ order Butterworth filter with a cut off frequency of 80 $\mathrm{Hz}$ is applied to $X(t)$. A low pass filter allows all frequencies below the cut off frequency to be passed and frequencies above to be blocked. An $80 \mathrm{~Hz}$ cut off frequency allows the signal to be played through the sound card and equipment used in the hertz project. To listen to the processed infrasound on a standard laptop speaker the cut off frequency of the final low pass filter can be adjusted to $300 \mathrm{~Hz}$. 


\section{A2: Equipment setup}

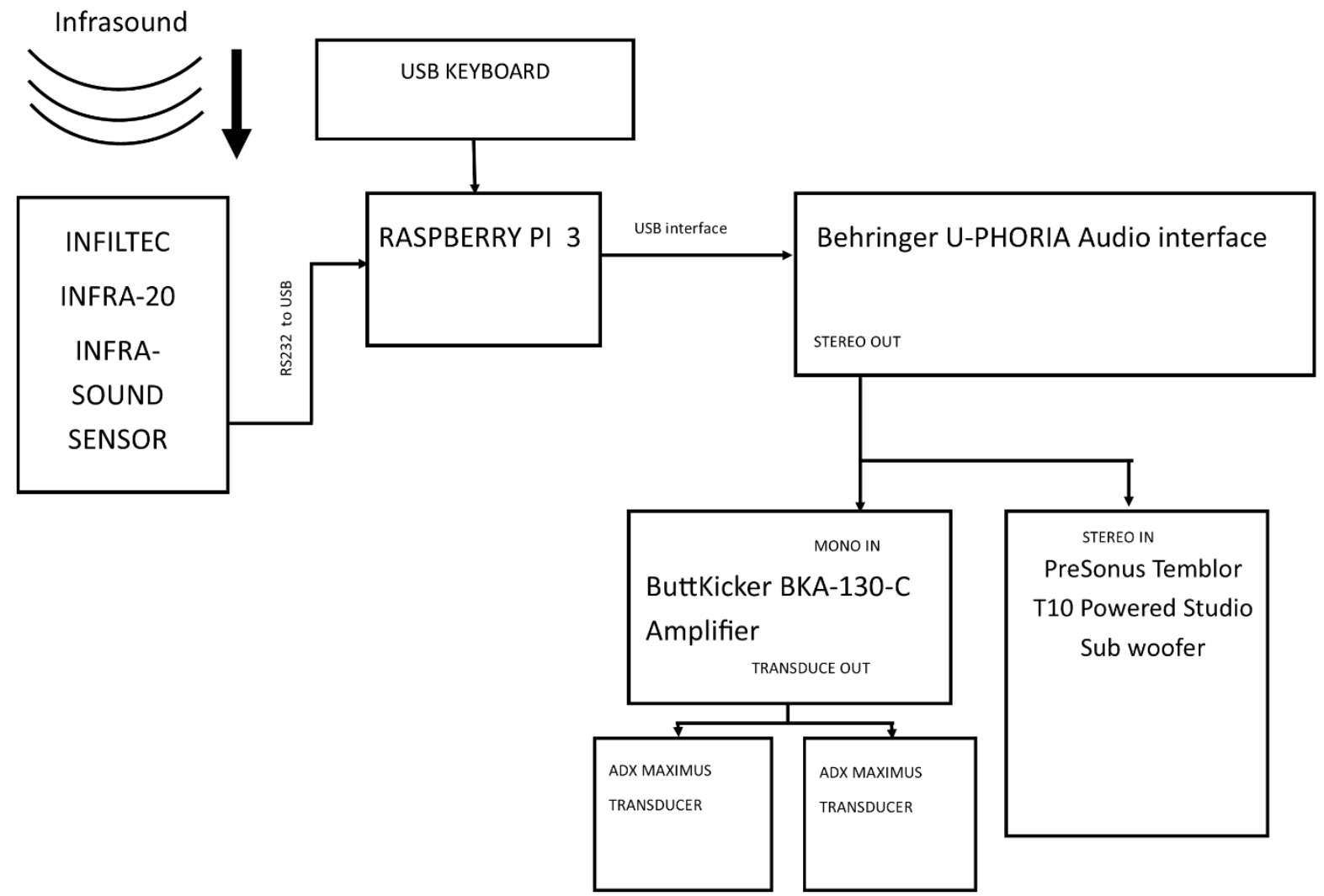

Figure S1: Flow diagram showing how the equipment was interfaced. Arrows indicate the direction of signal flow through the hertz system.

Figure S1 shows a box diagram of how the various pieces of equipment that made up hertz were interfaced. Arrows show the direction of signal flow through the system. The Raspberry PI performed the signal processing as described in section A1. The USB keyboard could be used to select processed real time infrasound signals recorded by the INFILTEC INFRA-20 sensor or from several pre-recorded infrasound samples

The Audio out from the Behringer U-PHORIA AUDIO interface was set at 100\% in the software. The individual audio settings on the Transducer and subwoofer were set at setup on location and this often depended on the location. The PreSonus trembler was rated at 250W. 